. LA-UR 909

TITLE: $\quad$ BLACK BOX MULTIGRID SOLVER FOR DEFINITE AND INDEFINITE PROBLEMS

RECEIVED

AUTHOR(S): $\quad$ Yair Shapira

JAN 21997

OSTI

SUBMITTED TO: $\quad$ External Distribution

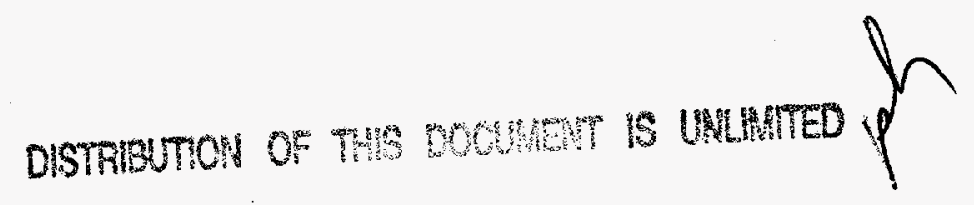

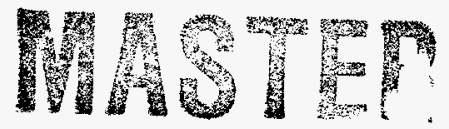

By acceptance of this article, the publisher recognizes that the U.S. Government retains a nonexclusive royalty-free license to publish or reproduce the published form of this contribution or to allow others to do so, for U.S. Government purposes. 


\section{DISCLAIMER}

This report was prepared as an account of work sponsored by an agency of the United States Government. Neither the United States Government nor any agency thereof, nor any of their employees, make any warranty, express or impliet, or assumes any legal liability or responsibility for the accuracy, completeness, or usefulness of any information, apparatus, product, or process disclosed, or represents that its use would not infringe privately owned rights. Reference herein to any specific commercial product, process, or service by trade name, trademark, manufacturer, or otherwise does not necessarily constitute or imply its endorsement, recommendation, or favoring by the United States Government or any agency thereof. The views and opinions of authors expressed herein do not necessar. ily state or reflect those of the United States Government or any agency thereof. 


\section{DISCLAIMER}

Portions of this document may be illegible in electronic image products. Images are produced from the best available original document. 


\title{
Black Box Multigrid Solver for Definite and Indefinite Problems
}

\author{
Yair Shapira \\ Computer Science Department, Technion, Haifa 32000, Israel \\ Now at Los Alamos National Laboratory \\ MS B-256, Los Alamos, NM 87545, e-mail: yairs@lanl.gov
}

\begin{abstract}
A two-level analysis method for certain separable problems is introduced. It motivates the definition of improved versions of Black Box Multigrid for diffusion problems with discontinuous coefficients and indefinite Helmholtz equations. For anisotropic problems, it helps in choosing suitable implementations for frequency decomposition multigrid methods. For highly indefinite problems, it provides a way to choose in advance a suitable mesh size for the coarsest grid used. Numerical experiments confirm the analysis and show the advantage of the present methods for several examples.
\end{abstract}

KEY WORDS elliptic PDEs, discontinuous coefficients, indefinite Helmholyz equations

\section{Preliminaries}

Consider the nonsingular linear system of equations

$$
A x=b,
$$

arising, for example, from a discretization of the elliptic PDE

$$
-\nabla(D \nabla u)+\vec{\kappa} \cdot \nabla u+\beta u=f
$$

in $\Omega \subset R^{d}$ with suitable boundary conditions, where $d$ is the dimension of the problem and $D, \vec{\kappa}$ and $\beta$ are given functions ( $D$ is a $d \times d$ symmetric and 
uniformly positive definite matrix and $\vec{\kappa}$ is a $d$-dimensional vector). Assume that $A$ is an operator in $V(\bar{\Omega})$, where $\bar{\Omega} \subset\left\{\vec{j}=\left(j_{1}, \ldots, j_{d}\right)\right\} \subset Z^{d}$ is the grid and, for any set $g \subset Z^{d}, V(g)$ is the linear space of functions defined on $g$. With this assumption, $A$ may be considered a tensor $A=\left(a_{\vec{i}, \vec{j}}\right)_{\vec{i}, \vec{j} \in \bar{\Omega}}$. In the following, we will treat $A$ both as a matrix and a tensor, assuming that the $\vec{i}$ th equation in the matrix $A$ (with some ordering) corresponds to grid point

$\vec{i}$. For $1 \leq j \leq d$, denote by $1^{(j)}$ the $j$ th column vector of the identity matrix of order $d$. Define

$$
\partial \bar{\Omega}=\left\{\vec{i} \in \bar{\Omega} \mid \exists j, 1 \leq j \leq d, \text { such that } \vec{i}+1^{(j)} \notin \bar{\Omega} \text { or } \vec{i}-1^{(j)} \notin \bar{\Omega}\right\} .
$$

When periodic boundary conditions are imposed, $\Omega$ and $\bar{\Omega}$ are torus and discrete torus, respectively. Hence in this case $\partial \bar{\Omega}=\emptyset$.

For any set $s$, let $|s|$ denote its intensity. For any tensor $M=\left(m_{\vec{i}, \vec{j}}\right)$, define the off-diagonal sum operator $r(M)$ by

$$
r_{\vec{i}}(M)=\sum_{\vec{j} \in \bar{\Omega}, \vec{j} \neq i} m_{\vec{i}, \vec{j}}
$$

\subsection{Definition of the Abstract Two-Level Method}

Let $\tilde{S}: x \rightarrow \tilde{S} x, \tilde{S} \equiv \tilde{S}(A, b)$, be a smoothing (relaxation) procedure for (1) with the corresponding iteration matrix $S$. Let $\nu_{1}$ and $\nu_{2}$ be positive integers denoting, respectively, the number of presmoothings and number of postsmoothings used. The operators $R$ (restriction), $P$ (prolongation) and $Q$ (coarse grid coefficient matrix) will be defined later. The abstract two-level (TL) procedure is defined by

$$
\operatorname{TL}\left(x_{i n}, A, b, x_{o u t}\right): \quad x_{o u t}=\tilde{S}^{\nu_{2}}\left(\tilde{S}^{\nu_{1}} x_{i n}+P Q^{-1} R\left(b-A \tilde{S}^{\nu_{1}} x_{i n}\right)\right) .
$$

An iterative application of TL is given by

$$
\begin{aligned}
& x_{0}=0, k=0 \\
& \text { while }\left\|A x_{k}-b\right\|_{2} \geq \text { threshold } \cdot\left\|A x_{0}-b\right\|_{2} \\
& \qquad \operatorname{TL}\left(x_{k}, A, b, x_{k+1}\right) \\
& \quad k \leftarrow k+1 \\
& \text { endwhile. }
\end{aligned}
$$

Note that the iteration matrix for this method is

$$
S^{\nu_{2}}\left(I-P Q^{-1} R A\right) S^{\nu_{1}}
$$

This representation is the basis for the two-level analysis in Section 3 below. 


\subsection{Definition of Black Box Multigrid}

Here we describe the Black Box Multigrid method of [5]. It is denoted in the sequel by $\mathrm{BBOX}$.

Assume that $A$ is of $3^{d}$-coefficient stencil, that is,

$$
|\vec{i}-\vec{j}|_{\infty}>1 \Rightarrow a_{\vec{i}, \vec{j}}=0 .
$$

For any integer $m$, denote ' $m$ is even' by $2 \mid m$ and ' $m$ is odd' by $2 \not \Varangle m$. For any index set $q \subset\{1, \ldots, d\}$, define the set

$$
g(q)=\left\{\vec{j} \in \bar{\Omega} \mid m \in q \Leftrightarrow 2 \nmid j_{m}\right\} .
$$

The family of disjoint sets $\{g(q)\}_{q \subset\{1, \ldots, d\}}$ may be thought of as a coloring of $\bar{\Omega}$ in which the set $g(q)$ corresponds to color $q$ (see [12], Method B). Typically, $g(\emptyset)$ serves as a coarse grid. In the sequel, we will also use the notation $c=g(\emptyset)$ and $f=\bar{\Omega} \backslash c$. This induces a block form for $A$ :

$$
A=\left(\begin{array}{cc}
A_{f f} & A_{f c} \\
A_{c f} & A_{c c}
\end{array}\right)
$$

where $A_{f f}$ and $A_{c c}$ are of order $|f|$ and $|c|$, respectively.

For any $\vec{i} \in \bar{\Omega}$, let $q(\vec{i}) \subset\{1, \ldots, d\}$ be the set for which $\vec{i} \in g(q(\vec{i}))$. For each $\vec{i} \in f$, define the sets

$$
t_{\vec{i}}=\left\{\vec{m} \in \bar{\Omega}|| \vec{m}-\left.\vec{i}\right|_{\infty} \leq 1, q(\vec{m}) \subset q(\vec{i})\right\}
$$

and, for each $\vec{j} \in t_{\vec{i}}$, the sets

$$
s_{\vec{i}, \vec{j}}=\left\{\vec{m} \in \bar{\Omega}|| \vec{m}-\left.\vec{i}\right|_{\infty} \leq 1, q(\vec{m}) \cap q(\vec{i}) \subset q(\vec{j}) \subset q(\vec{m})\right\} .
$$

For $\vec{i} \in c$, define $t_{\vec{i}}=s_{\vec{i}, \vec{i}}=\{\vec{i}\}$. In the prolongation, $t_{\vec{i}}$ is the set of grid points contributing to $\vec{i}$ and $s_{\vec{i}, \vec{j}}$ is the set of grid points on which a stencil sum ('collapse') is done to compute this contribution. Define the tensors $U=\left(u_{\vec{i}, \vec{j}}\right)$ and $L=\left(l_{\vec{i}, \vec{j}}\right)$ by

$$
\begin{aligned}
u_{\vec{i}, \vec{j}} & =\left\{\begin{array}{cc}
\sum_{\vec{m} \in s_{\overrightarrow{i j}}} \tilde{a}_{\vec{i}, \vec{m}} & \vec{j} \in t_{\vec{i}} \\
0 & \text { otherwise }
\end{array}\right. \\
\text { and } \quad l_{\vec{i}, \vec{j}} & =\left\{\begin{array}{cc}
\sum_{\vec{m} \in s_{\overrightarrow{j i}}} \tilde{a}_{\vec{m}, \vec{j}} & \vec{i} \in t_{\vec{j}}, \vec{i} \neq \vec{j} \\
0 & \text { otherwise, }
\end{array}\right.
\end{aligned}
$$

respectively, where (see [7])

$$
\tilde{a}_{\vec{i}, \vec{j}}=\left\{\begin{array}{cc}
-r_{\vec{i}}(A) & \vec{j}=\vec{i} \text { and }\left|a_{\vec{i}, \vec{i}}+r_{\vec{i}}(A)\right| \ll\left|a_{\vec{i}, \vec{i}}\right| \\
a_{\vec{i}, \vec{j}} & \text { otherwise. }
\end{array}\right.
$$


Assume that the variables are ordered in blocks corresponding to colors $q$ with decreasing order of $|q|$. Since $t_{\vec{i}} \subset\{\vec{i}\} \cup \cup_{|s|<|q(\vec{i})|} g(s), U$ and $L$ are upper and strictly lower triangular matrices, respectively. Define

$$
P=U^{-1} \operatorname{diag}(U) \text { and } R=\operatorname{diag}(U)(L+\operatorname{diag}(U))^{-1} .
$$

For symmetric problems, these definitions are equivalent to those of [5]; for nonsymmetric ones, however, they are slightly different from those recommended in [6]. We prefer the present definitions because they allow the simplifications in Section 2.4 below.

For any set $g \subset \bar{\Omega}$, let $J_{g}: V(\bar{\Omega}) \rightarrow V(g)$ be the injection

$$
\left(J_{g} v\right)_{\vec{j}}=v_{\vec{j}}, v \in V(\bar{\Omega}), \vec{j} \in g .
$$

The definition of $\mathrm{BBOX}$ is completed by

$$
\text { either } \begin{aligned}
Q^{-1} & =\left(\begin{array}{cc}
\operatorname{diag}\left(A_{f f}\right)^{-1}\left(R_{f f}\right)^{-1} & 0 \\
0 & \left(J_{c} R A P J_{c}^{t}\right)^{-1}
\end{array}\right) \\
\quad \text { or } Q^{-1} & =\left(\begin{array}{cc}
0 & 0 \\
0 & \left(J_{c} R A P J_{c}^{t}\right)^{-1}
\end{array}\right) .
\end{aligned}
$$

Approach (11) is the one used in [5], whereas (12) is used in [13].

\subsection{Difficulties with Black Box Multigrid}

We have found in our experiments that Black Box Multigrid has three sources of difficulties:

1. The examples tested in [2] [5] [6] use odd number of grid points in each spatial direction and coarse grids consisting of odd numbered variables of the next finer grid, so that coarse grids always include boundary points. When coarse grids consist of even numbered variables (as for the current implementation) the convergence is somewhat slower.

2. It was pointed out in [13] that Black Box Multigrid stagnates for certain diffusion problems with discontinuous coefficients. This phenomenon is due to strong coupling in the third-level coefficient matrix between subdomains which are only weakly coupled in the original system (see [2]). This strong coupling arises from the collapse (7)-(8) which mixes oblique connections with Cartesian ones, thus introduces strong connections between variables which should be only weakly connected. 
3. The performance of Black Box Multigrid deteriorates when (even small) amount of indefiniteness, namely, negative $\beta$ in (2), is inserted into the equation (by 'Black Box Multigrid' we refer here to the naive, straightforward extension of the method of [5] to indefinite problems (Section 1.2)).

To illustrate the second difficulty, consider Equation (2) in the 2-d region $(0,62) \times(0,62)$ with $\vec{\kappa} \equiv 0, \beta \equiv 0$ and $D=\operatorname{diag}(\tilde{D}, \tilde{D})$, where

$$
\tilde{D}(x, y)=\left\{\begin{array}{cc}
\frac{1000+1}{2} \cdot \frac{1}{1000} & (x, y) \in \theta \equiv\{(x, y)|| x-\omega|+| y-\omega \mid \leq 1\} \\
1000 & (x, y) \in(0, \omega) \times(0, \omega) \cup(\omega, 62) \times(\omega, 62) \backslash \theta \\
1 & (x, y) \in(0, \omega) \times(\omega, 62) \cup(\omega, 62) \times(0, \omega) \backslash \theta \\
0 & (x, y) \notin(0,62) \times(0,62),
\end{array}\right.
$$

and $0<\omega<62$ is the breaking point. The boundary conditions are

$$
\begin{array}{cc}
u_{n}=0 & x=0 \text { or } y=0 \\
\tilde{D} u_{n}+0.5 u=0 & x=62 \text { or } y=62 .
\end{array}
$$

The finite volume scheme of [2] is used over an $N \times N$ uniform grid with $N=63$ and the origin lying on the grid point numbered $(1,1)$. The coarse grids consist of even numbered points of the next finer grid (as defined in Section 1.2); this implementation is used throughout the paper, except for certain results in Table 2.1. A four-color Gauss-Seidel smoother ([1] and Method $\mathrm{A}$ in [12]) is used in a $\mathrm{V}(1,1)$ multigrid cycle. The convergence factor (cf) is defined in (26). It is seen from Table 1.3 that stagnation occurs when the breaking point $\omega$ lies on all grids.

Table 1: Convergence factors (cf) for BBOX for diffusion problems with discontinuous coefficients.

\begin{tabular}{|c|c||c|c|}
\hline$\omega$ & levels & $(11)$ & $(12)$ \\
\hline 30 & 2 & .050 & .075 \\
30 & 4 & .083 & .120 \\
31 & 2 & .067 & .096 \\
31 & 3 & .980 & .980 \\
\hline
\end{tabular}




\section{Improved Versions of Black Box Multigrid}

\subsection{Improved BBOX for Definite Problems}

First we introduce a version which avoids the first difficulty in Section 1.3. Our numerical experiments and the analysis in Section 2.4 suggest that the off-diagonal row-sums $-r_{\vec{i}}(A)$ in (9) should be used only when the prolongation is done along boundaries. This means that one should replace (9) by the following criterion:

$$
\tilde{a}_{\vec{i}, \vec{j}}=\left\{\begin{array}{cc}
-r_{\vec{i}}(A) & \vec{j}=\vec{i} \in f \text { and } t_{\vec{i}} \subset \partial \bar{\Omega} \\
a_{\vec{i}, \vec{j}} & \text { otherwise. }
\end{array}\right.
$$

Next, we treat the second difficulty of Black Box Multigrid. The algorithm introduced in [10] (also discovered by the authors of [2] in an earlier report) suggests to 'throw' off diagonal elements to the main diagonal; this is done by modifying the $\tilde{a}_{\vec{i}, \vec{j}}$ 's defined in (13) by

$$
\tilde{a}_{\vec{i}, \vec{j}} \leftarrow\left\{\begin{array}{cc}
\tilde{a}_{\vec{i}, \vec{i}}+\sum_{\vec{m} \in \bar{\Omega} \backslash \bar{t}_{\vec{i}} \backslash s_{\vec{i}, \vec{i}}} \tilde{a}_{\vec{i}, \vec{m}} & \vec{j}=\vec{i} \in f \\
0 & q(\vec{i}) \backslash q(\vec{j}) \neq \emptyset \neq q(\vec{j}) \backslash q(\vec{i}) \\
\tilde{a}_{\vec{i}, \vec{j}} & \text { otherwise }
\end{array}\right.
$$

(the main diagonal elements $\tilde{a}_{\vec{i}, \vec{i}}$ are modified first). The alternative approach suggested here 'throws' to the main diagonal only elements which are too large to participate in the collapse (7)-(8). Thus, the present version reads as follows. Define

$$
\hat{s}_{\vec{i}, \vec{j}}=\left\{\vec{m} \in s_{\vec{i}, \vec{j}}|| \tilde{a}_{\vec{i}, \vec{m}}|\leq \tau| \tilde{a}_{\vec{i}, \vec{j}} \mid\right\},
$$

where $\tau \geq 1$ is a parameter. Do (13) and then, instead of (7)-(8), do

$$
\begin{aligned}
& u_{\vec{i}, \vec{j}}=\left\{\begin{array}{cc}
\sum_{\vec{m} \in \hat{s}_{\vec{i}}} \tilde{a}_{\vec{i}, \vec{m}} & \vec{j} \in t_{\vec{i}}, j \neq i \\
\sum_{\vec{m} \in \hat{s}_{\vec{i} \vec{i}}} \tilde{a}_{\vec{i}, \vec{m}}+\sum_{\vec{k} \in t_{\vec{i}}} \sum_{\vec{m} \in s_{\vec{i} \vec{k}} \backslash \tilde{s}_{\vec{i} \vec{k}} \tilde{a}_{\vec{i}, \vec{m}}} & \vec{j}=\vec{i} \\
0 & \text { otherwise, }
\end{array}\right. \\
& l_{\vec{i}, \vec{j}}=\left\{\begin{array}{cc}
\sum_{\vec{m} \in \hat{s}_{\overrightarrow{j i}}} \tilde{a}_{\vec{m}, \vec{j}} & \vec{i} \in t_{\vec{j}}, j \neq i \\
0 & \text { otherwise. }
\end{array}\right.
\end{aligned}
$$

Typically, one should choose $\tau$ satisfying

$$
1<\tau<\min _{\vec{i}, \bar{j} \in \bar{\Omega},\left|a_{\vec{i}, \vec{j}}\right| \geq 4\left|a_{\vec{j}, \vec{j}}\right|}\left|a_{\vec{i}, \vec{i}}\right| /\left|a_{\vec{j}, \vec{j}}\right| .
$$

The improved results obtained with $\tau=10$ for the example in Section 1.3 are displayed in Table 2.1. In our numerical experiments, we also consider 
the 'staircase' problem (Example IV in [2]) with a $65 \times 65$ grid. For this grid, Criterion (13) suggests that the off-diagonal row-sums $-r_{\vec{i}}(A)$ should not be used on the first level (since the first coarse grid is interior to the finest one) but should be used in coarser levels on the upper and right edges of the discrete boundary, where coarse grid boundary points coincide with fine grid ones. The results in Table 2.1 show the advantage of BBOX with the modified collapse in comparison with the method of [10] (implemented with (11)) (it was also observed that multi level implementations of the method of [10] stagnate for the highly indefinite examples in Section 4).

Table 2: Convergence factors for BBOX with the modified collapse (with $\tau=$ 10). For comparison, results for the Kettler-Meijerink method are displayed in the last column.

\begin{tabular}{|c|c||c|c|c|}
\hline$\omega$ & levels & $(11)$ & $(12)$ & {$[10]$} \\
\hline 30 & 4 & .084 & .114 & .320 \\
31 & 4 & .082 & .129 & .111 \\
\hline staircase & 4 & .077 & .096 & .121 \\
\hline
\end{tabular}

We have also used the above staircase example (again with $N=65$ ) to show the advantage of the criterion (13). The coarse grids consist of either even numbered points or odd numbered points of the next finer grid. Note that Criterion (9) corresponds to either the first and third rows in Table 2.1 or the second and fourth ones, depending on the interpretation of the notation ' $\ll$ ' used there. Criterion (13), on the other hand, corresponds to the second and third rows in Table 2.1 (at least on the finest level; the results in the third row can be improved by using Criterion (13) on coarse levels also, see Table 2.1). Thus, it gives optimal results regardless of the construction of coarse grids.

Table 3: Various four-level implementations of BBOX with the modified collapse (with $\tau=10)$ to the staircase problem $(N=65)$.

\begin{tabular}{|c|c||c|}
\hline coarse grids consist of: & $\tilde{a}_{\vec{i}, \vec{i}}$ & convergence factor \\
\hline odd numbered points & $a_{\vec{i}, \vec{i}}$ & .161 \\
odd numbered points & $-r_{\vec{i}}(A)$ & .090 \\
\hline even numbered points & $a_{\vec{i}, \vec{i}}$ & .089 \\
even numbered points & $-r_{\vec{i}}(A)$ & .209 \\
\hline
\end{tabular}




\subsection{Improved BBOX for Indefinite Problems}

The idea is to modify the definition of the restriction and prolongation operators such that the contribution from the term $\beta u$ in (2) is distributed among spatial directions. To this end, we suggest two possible strategies. The first approach suggests to write $A=G+H$, where $H$ corresponds to a discretization of the term $\beta u$ in (2). Define the upper triangular matrices $U(G)$ and $U(H)$ by applying (7) to $G$ and $H$, respectively. Consider the prolongation to a point $\vec{i}$ along certain spatial directions. In order to distribute the contribution from $\beta$ properly, $U(H)_{\vec{i}, \vec{j}}$ should be multiplied by the relative diffusion along these directions, namely,

$$
U(H)_{\vec{i}, \vec{j}} \leftarrow U(H)_{\vec{i}, \vec{j}} \cdot r_{\vec{i}+\vec{e}(\vec{i})}(U(G)) / r_{\vec{i}+\vec{e}(\vec{i})}(G),
$$

where $\vec{e}(\vec{i})=2 \vec{g}(\vec{i})$ and $\vec{g}(\vec{i}) \in Z^{d}$ is the minimal integer vector (in, say, the $l_{1}$ norm) for which $\vec{i}+\vec{g}(\vec{i})$ and $\vec{i}+2 \vec{g}(\vec{i})$ are in $\bar{\Omega} \backslash \partial \bar{\Omega}$ ( $\vec{e}$ is introduced to avoid the effect of boundary conditions). Alternatively, one can define $\vec{e}(\vec{i}) \equiv 0$ and assume that the matrix argument of the operator $r(\cdot)$ is extended such that its stencil is defined on an infinite grid. $P$ is then obtained by setting $U=U(G)+U(H)$ and proceeding as in Section 1.2. $R$ is defined in a similar way. The lower right block matrix of $Q$ in (11)-(12) is given automatically in the decomposed form $J_{c}(R G P+R H P) J_{c}^{t}$, suitable for recursion in multi-level implementations.

The above definition requires larger storage and set-up time than that of standard BBOX. Furthermore, it requires the a-priori knowledge of the splitting $A=G+H$. Hence, we introduce an alternative approach, which coincides with the previous one for two-level implementations for $(2 d+1)$ coefficient stencils for the Helmholtz equation. It reads as follows. Define the discrete $\beta$ at point $\vec{i}$ by

$$
\tilde{\beta}_{\vec{i}}=a_{\vec{i}+\vec{e}(\vec{i}), \vec{i}+\vec{e}(\vec{i})}+r_{\vec{i}+\vec{e}(\vec{i})}(A) .
$$

Define the relative diffusion at point $\vec{i} \in f$ by

$$
d_{\vec{i}}=\frac{\sum_{\vec{j} \in t_{\vec{i}},|\vec{i}-\vec{j}|=1} \hat{a}_{\vec{i}, \vec{j}}}{\sum_{\vec{j} \in \bar{\Omega},|\vec{i}-\vec{j}|_{1}=1} \hat{a}_{\vec{i}, \vec{j}}} .
$$

Do (13) and then modify the resulting $\tilde{a}_{\vec{i}, \vec{j}}$ 's by

$$
\tilde{a}_{\vec{i}, \vec{j}} \leftarrow\left\{\begin{array}{cc}
\tilde{a}_{\vec{i}, \vec{i}}-\tilde{\beta}_{\vec{i}}\left(1_{\vec{i}}-d_{\vec{i}+\vec{e}(\hat{i})}\right) & \vec{i}=\vec{j} \in f \\
\tilde{a}_{\vec{i}, \vec{j}} & \text { otherwise }
\end{array}\right.
$$


where

$$
1_{\vec{i}}=\left\{\begin{array}{cc}
0 & \vec{i} \in f \text { and } t_{\vec{i}} \subset \partial \bar{\Omega} \\
1 & \text { otherwise. }
\end{array}\right.
$$

Thus, the contribution of the Helmholtz term $\beta u$ to main diagonal elements is multiplied by the amount of diffusion in the directions of prolongation (relatively to the total diffusion). (7) and (8) are then used to create $P$ and $R$ and $Q$ is defined as in (11)-(12). This approach works well in practice, e.g., for highly indefinite problems with variable coefficient $\beta$ and non-rectangular domains. It is denoted hereafter by Averaged Black Box multigrid (ABOX).

\subsection{Representations of $P$ for BBOX}

For any two sets $t_{2} \subset t_{1} \subset\{1, \ldots, d\}$, define

$$
P_{t_{1}, t_{2}}=\left\{\begin{array}{cc}
-J_{g\left(t_{1}\right)}^{t} J_{g\left(t_{1}\right)} P^{-1} J_{g\left(t_{2}\right)}^{t} J_{g\left(t_{2}\right)} & t_{1} \neq t_{2} \\
0 & t_{1}=t_{2}
\end{array}\right.
$$

For any ordered set $s$, let $\Pi_{s}$ denote a product of elements indexed in $s$ with a decreasing index order. Then we have

$$
P^{-1}=\Pi_{1 \leq j \leq d} \Pi_{\left|t_{2}\right|=d-j} \Pi_{t_{2} \subset t_{1}}\left(I-P_{t_{1}, t_{2}}\right)
$$

and, hence,

$$
\begin{aligned}
P & =\Pi_{0 \leq j \leq d-1} \Pi_{\left|t_{2}\right|=j} \Pi_{t_{2} \subset t_{1}}\left(I+P_{t_{1}, t_{2}}\right) \\
& =I+\sum_{k \geq 2, t_{1} \subset t_{2} \subset \cdots \subset t_{k}} \Pi_{1 \leq l \leq k-1} P_{t_{l+1}, t_{l}}
\end{aligned}
$$

This representation will be useful in the next section.

\subsection{Simplified Representations of $P$ for ABOX}

The motivation for the definition of ABOX lies in the opportunity to simplify further the representation of $P$ for schemes involving $(2 d+1)$-coefficient stencils, such as finite volume and finite difference schemes. In this case the coefficient matrix $A$ may be written in the form

$$
A=\sum_{i=1}^{d} X_{i}
$$

where $X_{i}$ represents a three-point discretization of the derivatives in the $i$ th spatial direction (including possible substitution for these derivatives at 
boundaries from boundary conditions). It is assumed that the term $\beta u$ in (2) contributes to $\operatorname{diag}(A)$ only; furthermore, it is assumed that the amount of this contribution at point $\vec{i} \in \bar{\Omega}$ is $\tilde{\beta}_{\vec{i}}$, out of which the amount

$$
\tilde{\beta}_{\vec{i}} \cdot r_{\vec{i}+\vec{e}(\vec{i})}\left(X_{k}\right) / r_{\vec{i}+\vec{e}(\vec{i})}(A)
$$

goes to $\left(X_{k}\right)_{\vec{i}, \vec{i}}(1 \leq k \leq d)$.

Assume also that for each $\vec{i} \in \partial \bar{\Omega}$ and $1 \leq j \leq d$

$$
\left\{\vec{i}+1^{(j)} \notin \bar{\Omega} \text { or } \vec{i}-1^{(j)} \notin \bar{\Omega}\right\} \Rightarrow \vec{i}_{j} \bmod 2 \in \mathcal{S}_{\vec{i}},
$$

where $1^{(j)}$ denotes the $j$ th column vector of the identity matrix of order $d$ and $\mathcal{S}_{\vec{i}}$ is either $\{0\}$ or $\{1\}$. (more accurately, this assumption is required only for directions $j$ for which boundary conditions other than Neumann are imposed). This means that

$$
\text { junction points of } \partial \bar{\Omega} \text { belong to } g(\{1, \ldots, d\}) \cup c \text {. }
$$

With these assumptions, a simple representation of $P$ is available, with which the two-level analysis in Section 3 below is much easier to implement.

For (16), AutoMUG is defined as follows. Let

$$
P_{i}=2 I-\operatorname{diag}\left(X_{i}\right)^{-1} X_{i} \text { and } R_{i}=2 I-X_{i} \operatorname{diag}\left(X_{i}\right)^{-1} .
$$

Let $\tilde{T}_{i}$ be the diagonal matrix which has the same row-sums as $P_{i}$ and define $T_{i}=\Pi_{j \neq i} \tilde{T}_{j}$. Define

$$
R=J_{c} \Pi_{1 \leq j \leq d} R_{j}, P=\Pi_{0 \leq j \leq d-1} P_{d-j} J_{\mathrm{c}}^{t} \text { and } Q=J_{c} \sum_{j=1}^{d} T_{j} R_{j} X_{j} P_{j} J_{\mathrm{c}}^{t} .
$$

We also consider the Frequency Decomposition Multigrid (FDM) method of [9] for the solution of highly anisotropic equations. When $X_{i}$ is considerably smaller than all the other $X_{j}$ 's it is suggested in [16] and [9] to use two coarse grid corrections: one with the standard bilinear prolongation and the other with a bilinear-like prolongation but with alternating signs. In [16] the same coarse grid is used for both corrections, whereas in [9] the coarse grid used for the latter correction is shifted so that odd numbered variables are used in the $i$ th spatial direction. This appears to be a considerable improvement (see Table 3.2 below). Here we consider the Child of FDM (CFDM) and the Grandchild of FDM (GFDM) of [8]; these are variants of BBOX (here they are implemented with (13) and (14)) for which $2^{d}$ coarse grid corrections are used, each of which uses transfer operators with signs alternating in some of the spatial directions. 
For $1 \leq i \leq d$, define

$$
p_{i}=\left\{\vec{j} \in \bar{\Omega}|2| j_{i}\right\} .
$$

Define $\tilde{p}_{i}=p_{i}$ for the implementation of [16] and $\tilde{p}_{i}=\bar{\Omega} \backslash p_{i}$ for that of [9]. For any set $t \subset\{1, \ldots, d\}$, substitute

$$
g(t) \leftarrow\left(\cap_{i \notin t} \tilde{p}_{i}\right) \cap\left(\cap_{i \in t}\left(\Omega \backslash \tilde{p}_{i}\right)\right)
$$

and set $c=g(\emptyset)$ and $f=\bar{\Omega} \backslash c$. Note that this is the same as before for $\mathrm{ABOX}$ and for the implementation of [16]. With this redefinition, (15) holds also for the implementation of [9], provided that $P$ is upper triangular when the variables are ordered in blocks $g(t)$ with decreasing order of $|t|$. Define

$$
\begin{aligned}
& P_{i}=\left(I-\operatorname{diag}\left(X_{i}\right)^{-1} X_{i}\right) J_{p_{i}}^{t} J_{p_{i}} \\
& P_{i}=\left\{\begin{array}{ccc}
\text { (a) } & \left(I-\operatorname{diag}\left(X_{i}\right)^{-1} X_{i}\right) J_{p_{i}}^{t} J_{p_{i}} & \text { for CFDM } \\
\text { or (b) } & P_{i}=\left(\operatorname{diag}\left(X_{i}\right)^{-1} X_{i}-I\right) J_{\tilde{p}_{i}}^{t} J_{\tilde{p}_{i}} &
\end{array}\right. \\
& P_{i}=\left\{\begin{array}{cc}
\text { (c) } & \left(I-\operatorname{diag}\left(X_{i}\right)^{-1} X_{i}\right) J_{p_{i}}^{t} J_{p_{i}} \\
\text { or (d) } & P_{i}=\Theta_{i}\left(\operatorname{diag}\left(X_{i}\right)^{-1} X_{i}-I\right) J_{\tilde{p}_{i}}^{t} J_{\tilde{p}_{i}}
\end{array} \quad\right. \text { for GFDM, }
\end{aligned}
$$

where $\Theta_{i}$ is a diagonal matrix measuring the strength of the diffusion in the $i$ th spatial direction: if (d) is used in more than one spatial direction then $\Theta_{i}=0$; else,

$$
\Theta_{i}=\operatorname{diag}\left(\max \left(0,1-(d-1) r_{\vec{j}+\vec{e}(\vec{j})}\left(X_{i}\right) / r_{\vec{j}+\vec{e}(\vec{j})}\left(A-X_{i}\right)\right)\right)_{\vec{j} \in \bar{\Omega}} .
$$

Finally, we consider what we call $\operatorname{CFDM}\left(x_{i}\right)$, for which at most two coarse grid corrections are used, namely, Definition (b) is used only when $X_{i}$ is considerably smaller than all the other $X_{j}$ 's.

With the terminology of Section 2.3, (16) implies that $P_{t_{1}, t_{2}}=0$ if $\left|t_{1}\right|$ $t_{2} \mid \geq 2$. Consequently, (15) obtains the form

$$
\begin{aligned}
P= & I+\sum_{t \subset\{1, \ldots, d\},} \sum_{k \geq 1,} \sum_{1 \leq r_{1} \neq \cdots \neq r_{k} \leq d, r_{1}, \ldots, r_{k} \notin t} \Pi_{0 \leq l \leq k-1} P_{t \cup\left\{r_{1}, \ldots, r_{l+1}\right\}, t \cup\left\{r_{1}, \ldots, r_{l}\right\}} \\
= & \left.I+\sum_{t \subset\{1, \ldots, d\}, k \geq 1,1 \leq r_{1} \neq \cdots \neq r_{k} \leq d, r_{1}, \ldots, r_{k} \notin t}{ }^{t+1} \Pi_{0 \leq l \leq k-1}\left(\operatorname{diag}\left(\sum_{i \in t} X_{i}+\sum_{i=1}^{l+1} X_{r_{i}}\right)\right)^{-1} \operatorname{diag}\left(X_{r_{l+1}}\right) P_{r_{l+1}}\right) J_{g(t)}^{t} J_{g(t)} .
\end{aligned}
$$

In the remainder of this paper we consider the implementation (12). Note that for this approach one may substitute

$$
P \leftarrow P J_{c}^{t}, \quad R \leftarrow J_{c} R \text { and } Q \leftarrow R A P
$$


(in this order). The above equality is then reduced to

$$
P=J_{c}^{t}+\sum_{k \geq 1,1 \leq r_{1} \neq \cdots \neq r_{k} \leq d}\left(\Pi_{0 \leq l \leq k-1}\left(\operatorname{diag}\left(\sum_{i=1}^{l+1} X_{r_{i}}\right)\right)^{-1} \operatorname{diag}\left(X_{r_{l+1}}\right) P_{r_{l+1}}\right) J_{c}^{t} .
$$

If, in addition, the problem is separable, namely, the $\operatorname{diag}\left(X_{i}\right)^{-1} X_{i}$ 's commute with each other, then this simplifies to read (the proof is by induction on $d$, see [11])

$$
\begin{aligned}
P & =J_{c}^{t}+\sum_{k \geq 1,1 \leq r_{1}<\cdots<r_{k} \leq d}\left(\Pi_{0 \leq l \leq k-1} P_{r_{l+1}}\right) J_{c}^{t} \\
& =\Pi_{1 \leq i \leq d}\left(I+P_{i}\right) J_{c}^{t} .
\end{aligned}
$$

Note that $R$ also is equal to the right hand side of (21), provided that the multiplication by $J_{c}^{t}$ on the right is replaced by a multiplication by $J_{c}$ on the left and the $P_{i}$ 's are replaced by suitable $R_{i}$ 's. For ABOX, for example, one should use $R_{i}=J_{p_{i}}^{t} J_{p_{i}}\left(I-X_{i} \operatorname{diag}\left(X_{i}\right)^{-1}\right)$ and proceed as above, and similarly for the other methods. This gives a uniform representation for ABOX, CFDM, GFDM and AutoMUG and considerably simplifies the twolevel analysis introduced next.

\section{Two-Level Analysis for Black Box Multi- grid}

\subsection{The Two-Level Analysis Method}

Assume that $A$ is diagonalizable and that, for every eigenvector of $A$ of the form $v=\left\{v_{\vec{j}}\right\}_{\vec{j} \in \bar{\Omega}}$,

$$
v^{(\alpha)} \equiv\left\{(-1)^{\sum_{i=1}^{d} \alpha_{i} j_{i}} v_{\vec{j}}\right\}_{\vec{j} \in \bar{\Omega}}, \quad \alpha \in\{0,1\}^{d}
$$

are also eigenvectors of $A$ with the corresponding eigenvalues $\lambda^{(\alpha)}$. This assumption holds, for example, for (a) periodic problems with constant coefficients (namely, when $A$ is a circulant Toeplitz tensor), (b) problems with constant coefficients and $3^{d}$-coefficient stencils and (c) problems of the form (16) (see [17], Sec. 7.1). Let us compute the symbol $\hat{A}$ of $A$, namely, the representation of $A$ in the subspace spanned by the $v^{(\alpha)}$ 's. The basis used for this is $\left\{J_{g(s)}^{t} J_{g(s)} v\right\}_{s \subset\{1, \ldots, d\}}$. For any set $s$, let $2^{s}$ denote the family of subsets of $s$. Define the isomorphisms

$$
\begin{array}{cll}
s \in 2^{\{1, \ldots, d\}} \rightarrow \alpha(s) \in\{0,1\}^{d} & \text { by } \quad \alpha(s)_{i}=1 & \Leftrightarrow i \in s \\
\text { and } \operatorname{span}\left\{J_{g(s)}^{t} J_{g(s)} v\right\}_{s \subset\{1, \ldots, d\}} \rightarrow V\left(\{0,1\}^{d}\right) & \text { by } \quad J_{g(s)}^{t} J_{g(s)} v & \rightarrow \alpha(s) .
\end{array}
$$


Then the symbol $\hat{J}_{g(s)}$ is the $\left(\sum_{i=1}^{d} \alpha(s)_{i} 2^{i}+1\right)$ st row of the identity matrix of order $2^{d}$ and $\hat{J}_{p_{i}}=\sum_{s, i \notin s} \hat{J}_{g(s)}$. Define the symmetric orthogonal discrete Haar transform by

$$
H=\left(h_{\gamma, \delta}\right)_{\gamma, \delta \in\{0,1\}^{d}}, \quad h_{\gamma, \delta}=2^{-d / 2}(-1)^{\sum_{i=1}^{d} \gamma_{i} \delta_{i}}
$$

Then we have

$$
\hat{A}=H \operatorname{diag}\left(\lambda^{(\alpha)}\right)_{\alpha \in\{0,1\}^{d}} H .
$$

Clearly, for cases (a) and (b) $\hat{R}$ and $\hat{P}$ are also available. For (16), assume that the $X_{i}$ 's have constant main diagonals and commute with each other. Then the $\hat{X}_{i}$ 's can be computed the same way $\hat{A}$ was and $\hat{R}$ and $\hat{P}$ result from (21) (for GFDM it is also required that the $\Theta_{i}$ 's are multiples of the identity). The symbol of $Q$ is then obtained from the symbol product $\hat{Q}=\hat{R} \hat{A} \hat{P}$. For AutoMUG, the symbols of the restriction, prolongation and coarse grid coefficient matrix are computed similarly, using the definitions (18)-(19) and assuming that the matrices $J_{c} T_{i} J_{c}^{t}$ used there are multiples of the identity (see [11]).

Let $0 \leq k \leq d-1$ be a fixed integer and consider a $2^{d-k}$-color hyperplane relaxation with $k$-dimensional hyperplanes (for example, when $k=0$ this is a multi-color point relaxation with the colors $g(s), s \subset\{1, \ldots, d\})$. For the symbol $\hat{S}$ of this relaxation, write $\hat{A}=\left(\hat{A}_{i, j}\right)_{1 \leq i, j \leq 2^{d-k}}$, where the $\hat{A}_{i, j}$ 's are blocks of order $2^{k}$ corresponding to the various colors. The square matrix symbol of the first color relaxation is then given by

$$
\hat{S}_{1}=\left(\begin{array}{cccc}
0 & -\hat{A}_{1,1}^{-1} \hat{A}_{1,2} & \cdots & -\hat{A}_{1,1}^{-1} \hat{A}_{1,2^{d-k}} \\
0 & I &
\end{array}\right)
$$

and $\hat{S}$ is just the product of such symbols. The symbol of the iteration matrix (5) is then obtained by replacing individual matrices there by their corresponding symbols. For the multiple coarse grid correction methods CFDM and GFDM, two possible implementations exist: the additive approach, for which the iteration matrix symbol is

$$
\hat{S}^{\nu_{2}}\left(\hat{I}-\left(\sum_{j} \hat{P}_{j} \hat{Q}_{j}^{-1} \hat{R}_{j}\right) \hat{A}\right) \hat{S}^{\nu_{1}}
$$

(where $j$ runs over all coarse grid corrections) and a multiplicative approach, for which the residual is recomputed after every coarse grid term addition and the iteration matrix symbol is

$$
\hat{S}^{\nu_{2}} \Pi_{j}\left(\hat{I}-\hat{P}_{j} \hat{Q}_{j}^{-1} \hat{R}_{j} \hat{A}\right) \hat{S}^{\nu_{1}} .
$$


The spectrum of the iteration matrix may be computed by scanning over eigenvalues corresponding to elements in a set $V$ of eigenvectors of $A$ satisfying $|V| \geq\left\lceil|\bar{\Omega}| / 2^{d}\right\rceil$ and $u, v \in V \Rightarrow u \notin \operatorname{span}\left\{v^{(\alpha)}\right\}_{\alpha \in\{0,1\}^{d}}$ and computing numerically the spectra of the corresponding symbols of the iteration matrix.

\subsection{Applications}

For separable problems of the form (16) the spectrum of $A$ is the sum of those of the tridiagonal matrices $X_{i}$. In some cases it is known that these spectra lie in the interior of certain ellipses (or circles, using Gershgorin's theorem) in the complex plane; since the eigenvalues of the iteration matrix are given as a meromorphic function of those of the $X_{i}$ 's, one may scan over the corresponding ellipses (and possible poles of this function) and obtain an upper bound for the asymptotic convergence factor. Alternatively, the spectra of the $X_{i}$ 's can be computed numerically either by an LU method or by a Lanczos type method. Here we apply the two-level analysis method to some model problems for which these spectra are known in advance, namely, Dirichlet problems with constant coefficients in the unit square. The first example is the anisotropic diffusion equation

$$
-u_{x x}-\delta u_{y y}=f
$$

with a uniform $N \times N$ grid and the usual five-coefficient second order discretization. The red-black Gauss-Seidel (RB) smoother, (12), (14), $\nu_{1}=\nu_{2}=$ 1 and $N=63$ are used. It is well-known that for anisotropic problems $\mathrm{RB}$ is not an appropriate smoother for single coarse grid correction algorithms. From Table 3.2, however, it can be seen that it is quite efficient in the multiple coarse grid correction algorithms CFDM and GFDM, provided that the implementation of [9] is used. Note that the results for this implementation are only approximations for the actual convergence factors of the methods of [8], since (17) cannot hold for all the coarse grids simultaneously.

Next, we consider indefinite Helmholtz equations. For the slightly indefinite equation

$$
-u_{x x}-u_{y y}-20 u=f
$$

we obtained spectral radii similar to those for the Poisson equation, namely, .0625 for $\mathrm{ABOX}$ and .0741 for AutoMUG. This is in agreement with results in [13] (see Theorem 1 there and the discussion proceeding it). Then we turn to the more difficult highly indefinite equation

$$
-u_{x x}-u_{y y}-790 u=f .
$$


Table 4: Spectral radii of two-level iteration matrices for an anisotropic diffusion equation with Dirichlet boundary conditions. $N=63$, the RB smoother and $\nu_{1}=\nu_{2}=1$ are used.

\begin{tabular}{|c|c||c|c|c||c|c|c|}
\hline$\delta$ & residuals & {$[16]$} & {$[16]$} & {$[16]$} & {$[9]$} & {$[9]$} & {$[9]$} \\
\hline & & CFDM $(y)$ & CFDM & GFDM & CFDM $(y)$ & CFDM & GFDM \\
\hline $10^{-2}$ & not recomputed (22) & .967 & .956 & .967 & .281 & .281 & .274 \\
$10^{-2}$ & recomputed (23) & .956 & .956 & .956 & .081 & .084 & .077 \\
\hline $10^{-5}$ & not recomputed (22) & .995 & .995 & .995 & .003 & .052 & .002 \\
$10^{-5}$ & recomputed (23) & .995 & .995 & .995 & .000 & .083 & .001 \\
\hline
\end{tabular}

The (scaled) coefficient matrix for this problem with $N=15,31$ and 63 has (respectively) 38, 32 and 30 distinct negative eigenvalues, the five smallest (in magnitude) of which are displayed in the 1st, 4th and 7th columns of Table 3.2. This information shows that the problem is nearly singular in the sense of [4]. In the other columns of Table 3.2, the five largest (in magnitude) eigenvalues of the iteration matrices of $\mathrm{ABOX}$ and AutoMUG are displayed.

Although the basic iteration (4) diverges, it is seen that for $N=31$ and $N=63$ there exist only few isolated eigenvalues of magnitude larger than or close to one. Consequently, it is expected that a Lanczos-type acceleration method applied to (4) will yield suitable linear combinations of iterants in such a way that error components corresponding to large eigenvalues are annihilated. This results in convergence controlled by the small eigenvalues alone, as confirmed numerically in Section 4. Moreover, the analysis in [3] and the multi-level analysis in [13] show that the number of levels may be arbitrarily enlarged, provided that the appropriate mesh-size for the coarsest grid is unchanged. A $31 \times 31$ or a $15 \times 15$ grid is thus suitable for serving as a coarsest grid in a multi-level implementation for this problem.

\section{$4 \quad$ Numerical Results}

The numerical experiments presented here confirm the results of Section 3.2. As a matter of fact, the results in Table 4 coincide with those of Tables 3.2. When acceleration is used, the efficiency of $\mathrm{ABOX}$ for highly indefinite problems is shown. Due to its inexpensive multi-level implementation, AutoMUG appears to be competitive in some cases; however, it should be kept in mind that AutoMUG is less robust in the sense that it cannot handle stencils with more than $2 d+1$ coefficients. 
Table 5: Five largest negative eigenvalues of $A$ (scaled) and five largest (in magnitude) eigenvalues of two-level iteration matrices for the highly indefinite Helmholtz equation. The RB smoother and $\nu_{1}=\nu_{2}=1$ are used.

\begin{tabular}{|c|c|c||c|c|c||c|c|c|}
\hline$N=15$ & $N=15$ & $N=15$ & $N=31$ & $N=31$ & $N=31$ & $N=63$ & $N=63$ & $N=63$ \\
\hline$A$ & ABOX & AMUG & $A$ & ABOX & AMUG & $A$ & ABOX & AMUG \\
\hline-0.241 & 174.13 & -886.52 & $-1.14 \cdot 10^{-3}$ & -6.710 & -17.56 & $-1.16 \cdot 10^{-3}$ & -3.409 & 1.478 \\
-0.368 & 119.77 & -727.10 & $-1.90 \cdot 10^{-2}$ & 1.680 & 2.91 & $-8.42 \cdot 10^{-3}$ & 0.751 & -0.928 \\
-0.431 & 61.56 & -594.85 & $-2.07 \cdot 10^{-2}$ & 1.028 & -1.75 & $-9.97 \cdot 10^{-3}$ & 0.329 & 0.451 \\
-0.528 & -42.44 & -511.42 & $-5.04 \cdot 10^{-2}$ & -0.700 & 0.981 & $-1.08 \cdot 10^{-2}$ & 0.152 & -0.183 \\
-0.617 & -33.37 & -416.42 & $-6.03 \cdot 10^{-2}$ & 0.554 & 0.786 & $-1.63 \cdot 10^{-2}$ & -0.136 & -0.136 \\
\hline
\end{tabular}

We consider Equation (25) with Dirichlet boundary conditions. Although it is ill-posed, it is suitable for serving as a test problem for more realistic situations. It is discretized via a second-order five-point difference scheme on a uniform $N \times N$ grid. The initial guess is random in $(0,1)$. The solution is $u=0$. The RB smoother is used in $\mathrm{V}(0,1)$ or $\mathrm{V}(1,1)$-cycles. (12) and (14) are used. The second level equation is solved with six orders of magnitude accuracy.

In order to handle iteration matrix eigenvalues of magnitude larger than one, we apply to the basic two-level iteration (4) the Conjugate Gradients Squared (CGS) acceleration method of [14]. We define the following measures of efficiency: the convergence factor

$$
\mathrm{cf}=\frac{\left\|A x_{\text {last }}-b\right\|_{2}}{\left\|A x_{\text {last }-1}-b\right\|_{2}}
$$

and the preconditioned convergence factor

$$
\mathrm{pcf}=\left(\frac{\left\|\mathcal{P}^{-1}\left(A x_{\text {last }}-b\right)\right\|_{2}}{\left\|\mathcal{P}^{-1}\left(A x_{0}-b\right)\right\|_{2}}\right)^{2 /\left(\text { last } \cdot\left(\nu_{1}+\nu_{2}+1\right)\right)},
$$

where $\mathcal{P}$ is the multigrid preconditioner used and last is large enough to realize the process behavior (for pef, last is the first integer for which the $l_{2}$ norm of the preconditioned residual is reduced by six orders of magnitude). The preconditioned residual norm is available in the CGS process; furthermore, since the preconditioned system is better conditioned than the original one, the norm of the preconditioned residual is a better convergence measure than that of the residual itself. It was also checked that the $l_{2}$ and $l_{\infty}$ norms of the 
error decrease by at least four orders of magnitude during the convergence process.

The above definition of pcf takes into account the additional work required for smoothing and residual computation; its basic measure is the averaged convergence factor for a $\mathrm{V}(0,1)$-cycle.

In all our tests, we have found essentially no difference between the performances of (11) and (12) for indefinite problems. Since the latter is less expensive in terms of time and storage it was used here. The results are summarized in Tables 4 and 4.

Table 6: Convergence factors (cf) for two-level implementations for the highly indefinite equation. No acceleration is used.

\begin{tabular}{|c|c|c|c|c|c|}
\hline$N$ & $\nu_{1}$ & $\nu_{2}$ & BBOX & AutoMUG & ABOX \\
\hline 31 & 1 & 1 & 10.613 & 17.563 & 6.710 \\
\hline 63 & 1 & 1 & 2.007 & 1.478 & 3.409 \\
\hline
\end{tabular}

Table 7: Preconditioned convergence factors (pcf) for two-level implementations for the highly indefinite equation. CGS acceleration is used.

\begin{tabular}{|c|c|c||c|c|c|}
\hline$N$ & $\nu_{1}$ & $\nu_{2}$ & BBOX & AutoMUG & ABOX \\
\hline 31 & 1 & 1 & .976 & .798 & .784 \\
\hline 31 & 0 & 1 & .915 & .726 & .767 \\
\hline
\end{tabular}

Finally we consider four-level implementations for the slightly indefinite equation (24) with Dirichlet boundary conditions. The RB smoother is used for AutoMUG and a four-color Gauss-Seidel smoother ([1] and Method A in [12]) is used for the other methods. ABOX is implemented with (14). No acceleration is used. The advantage of $A B O X$ and AutoMUG is evident from Table 4. It is also seen that (11) is not better than (12).

\section{References}

[1] L.M. Adams and H.F. Jordan, Is SOR Color-Blind? SIAM J. Sci. Stat. Comput., 7 (1986), 490-506. 
Table 8: Convergence factors (cf) for four-level methods for the slightly indefinite equation. No acceleration is used.

\begin{tabular}{|c|c|c||c|c|c|c|c|}
\hline & & & $(11)$ & $(11)$ & $(12)$ & $(12)$ & \\
\hline$N$ & $\nu_{1}$ & $\nu_{2}$ & BBOX & ABOX & BBOX & ABOX & AutoMUG \\
\hline 31 & 1 & 1 & 7.196 & 0.077 & 7.195 & 0.063 & 0.131 \\
\hline 63 & 1 & 1 & 0.431 & 0.064 & 0.431 & 0.064 & 0.096 \\
\hline
\end{tabular}

[2] R. Alcouffe, A. Brandt, J.E. Dendy, and J. Painter, The Multigrid Method for the Diffusion Equation with Strongly Discontinuous Coefficients, SIAM J. Sci. Stat. Comput., 2 (1981), 430-454.

[3] J.H. Bramble, Z. Leyk and J.E. Pasciak, Iterative Schemes for NonSymmetric and Indefinite Elliptic Boundary Value Problems, Math. Comp., 60 (1993), 1-22.

[4] A. Brandt and S. Ta'asan, Multigrid methods for nearly singular and slightly indefinite problems. In W. Hackbusch and U. Trottenberg (eds.) Multigrid Methods II, Lecture Notes in Mathematics 1228, Springer-Verlag, Cologne, 1985, 100-122.

[5] J.E. Dendy, Black Box Multigrid, J. Comp. Phys., 48 (1982), 366-386.

[6] J.E. Dendy, Black Box Multigrid for Nonsymmetric Problems, Appl. Math. Comp., 13 (1983), 261-283.

[7] J.E. Dendy, Two Multigrid Methods for the Three-Dimensional Problems with Discontinuous and Anisotropic Coefficients, SIAM J. Sci. Stat. Comput., 8 (1987), 673-685.

[8] J.E. Dendy and C.C. Tazartes, Grandchild of The Frequency Decomposition Multigrid Method, SIAM J. Sci. Comput., 16 (1995), 307-319.

[9] W. Hackbusch, The Frequency Decomposition Multigrid Method, Part 1: Application to anisotropic equations, Numer. Math., 56 (1989), 229-245.

[10] R. Kettler and J.A. Meijerink, A Multigrid Method and a Combined Multigrid-Conjugate Gradient Method for Elliptic Problems with Strongly Discontinuous Coefficients. In General Domains, Shell Publ. 604, KSELP, Rijswijk, The Netherlands, 1981. 
[11] Y. Shapira, Two-Level Analysis of Automatic Multigrid for NonNormal and Indefinite Problems. Technical Report \#824 (revised version), Computer Science Department, Technion, Israel, July 1994.

[12] Y. Shapira, Coloring Update Methods. Technical Report \#851 (revised version), Computer Science Department, Technion, Israel, Sept. 1995, submitted to J. Comput. Appl. Math..

[13] Y. Shapira, Multigrid Techniques for Highly Indefinite Equations. In S.F. McCormick and T.A. Manteuffel (eds.) 8th Copper Mountain Conference on Multigrid Methods, NASA - Langley Research Center, Hampton, VA, 1995 (in press).

[14] P. Sonneveld, CGS, a Fast Lanczos-Type Solver for Nonsymmetric Linear Systems, SIAM J. Sci. Statist. Comput., 10 (1989), 36-52.

[15] K. Stuben and U. Trottenberg, Multigrid Methods: Fundamental Algorithms, Model Problem Analysis and Applications. In W. Hackbusch and U. Trottenberg (eds.) Multigrid Methods, Lecture Notes in Mathematics 960, Springer-Verlag, Berlin, 1982, 1-176.

[16] S. Ta'asan, Multigrid Methods for Highly Oscillatory Problems, Ph.D. Thesis, Weizmann Institute of Science, Rehovot, Israel, 1984.

[17] D. Young, Iterative Solution of Large Linear Systems, Academic Press, N.Y., 1971. 